\title{
Massive multi-agent systems control
}

\author{
Jean-Charles CAMPAGNE ${ }^{1}$, Alain CARDON $^{1,2}$, Etienne COLLOMB $^{3}$, and \\ Toyoaki NISHIDA ${ }^{3}$ \\ 1 LIP6 - UPMC - CNRS - 8, rue du Capitaine Scott - 75015 Paris - France \\ \{jean-charles.campagne, alain.cardon\} 01 lip6.fr \\ 2 IRD, Centre Ile-de-France - 32, rue Varagnat - 93143 Bondy Cedex - France \\ 3 The University of Tokyo - 7-3-1 Hongo, Bunkyo-ku, Tokyo 113-8656, Japan \\ \{etienne,nishida\}okc.t.u-tokyo.ac.jp
}

\begin{abstract}
In order to build massive multi-agent systems, considered as complex and dynamic systems, one needs a method to analyze and control the system. We suggest an approach using morphology to represent and control the state of large organizations composed of a great number of light software agents. Morphology is understood as representing the state of the multi-agent system as shapes in an abstract geometrical space, this notion is close to the notion of phase space in physics.
\end{abstract}

\section{Introduction}

With the advent of new computer technologies new large-scale systems are now possible. However, methods for actually building such complex system are less frequently proposed. Existing common approaches include : "manual tuning", emergence-based theory approaches, genetic approaches.

Manual tuning is only feasible for a couple of agents. It is impracticable for bigger organizations.

Emergence-based theories seek the understanding of the requirements at the microscopic level (the agent) in the hope that the macroscopic (the system) level will eventually behave appropriately. Many of these theories suggest that the agents composing the system have to be cooperative : resolving local conflict is sufficient to yield a proper global behavior ( $\mathrm{eg}[3]$ ). This hypothesis seems too restrictive [2] ; natural self-adaptive systems composed of many entities are not all locally-cooperative.

Agent genetic approaches, which include non-necessarily cooperative agents (eg [5]), seem to be promising. However, they lack of the ability of anaiyzing and understanding the system. It is difficult to understand how the system works by onity relying on the fitness function.

In order to build such a system one has to be able to analyze, maintain and control the behavior of the system. Deep understanding of the system workings is needed. And for such a system to be auto-adaptive, it needs to observe, analyze and control itself [4]. 
Our proposal For a system to be self-regulated, it has to have the ability to consider its internal state. We propose a way of describing the state of the agent organization in a problem-independent manner, by projecting the state of the agent organizations in an abstract geometrical space from various measurements made at the agent level (this is similar to the approach in physics as with phasespace), and letting the system access this representation in order to control itself. The underlying hypothesis is that the shapes representing the system's state are correlated to the system's behavior.

We describe the model, highlighting the important points, and then present an example of application of such an architecture applied to agent population control. We also discuss the advantages and current limitations based on the experiments with the implemented model.

\section{Description of the approach}

\subsection{Hypothesis}

We seek to correlate the micro-level behavior (agent) with the macro-level behavior (organization) using a generic approach (morphology). The hypothesis is that the shapes should be correlated to the system's behavior, and that it is possible to attract the system toward another state using the morphological description if the system fails to behave appropriately.

\subsection{General Description}

The system is composed of three main organizations : the aspectual organization that represents a phenomena; the morphological organization which describes the state of the aspectual organization in a geometrical way; and the analysis organization controlling the aspectual organization relying on the description given by the morphological organization and following the guidelines provided by the system designer. A more detailed description can be found in [1].

\subsection{Aspectual organization}

The aspectual organization, composed of many agents, represents a phenomena we want to study. This is the organization we seek to analyze and control. The term "aspectual" comes from the original agentification method proposed in [1].

In order to evaluate the system's state, the aspectual agents compute a value, called the "aspectual vector", as they run. This vector is a collection of values describing the agent's organizational state and its activity. The exact nature of these measures depend on the structure of the agent. 


\subsection{Morphological organization}

The whole collection of aspectual vectors make up the aspectual landscape of the aspectual organization which is then analyzed by the morphological agents.

Morphological agents attempt to describe what is happening in the aspectual organization in a geometrical way. The description does not take into account the ontology previously established : there is no semantics in the morphology space. Morphology space is only concerned with the activity and the organizational state of the agents. It points out structure, shapes, recurrent features, similarities, oppositions, dominant or recessive features... If we consider the aspectual measure as a mapping from a subset of the agent organizational state space to a numerical space (possibly multi-dimensional) ; the reciprocal is a function that modifies the agent behavior according to some target value so that the resulting aspectual vector of the agent would conform to that target value.

\subsection{Analysis organization}

By using a proper way of computing the morphology, the shapes revealed by the morphology are correlated to the system's behavior. We intend to exploit this correlation.

The analysis agents use the morphological description to examine the aspectual organization and to orientate the system accordingly to some generic guidelines instructed by the designer (for example : "global variable $\mathrm{X}$ of the system should be around value $\mathrm{Y}^{\prime \prime}$...). This is achieved by classifying and learning the morphology : as the system runs, typical shapes in the morphological spaces are revealed, these shapes are correlated to the system's behavior and categorized appropriately. Analysis agents can, following the designer's guidelines, influence the aspectual organization, either by direct injunctions on it, or by selecting appropriate shapes (learned from the system's past activity) and telling the morphological agents that this particular shape would be more appropriate than the current shape.

\section{Example}

We have developed an example using this approach in the context of agent population size control. The goal of this example is to illustrate how the global behavior of the system is correlated with its morphological description and how it is possible to exploit this correlation to control the system.

\subsection{Aspectual agents}

The aspectual organization is subjected to population control. Aspectual agents reside in a common environment where they "see" each other and from which they can extract some "energy" in order to survive.

Agents have some limited social skills : an agent can ask another agent to give it some energy. The asked agent can either cooperate or refuse. In the event 
of refusal, the asker "fights" the non cooperative agent. A fight results in the loss of energy from both antagonists, however the initiator of the fight looses less energy than the other one (simulating the benefit of initiating the attack).

If the energy level of an agent drops below zero, the agent "dies" and is removed from the organization. If an agent collects enough energy it can clone, yielding another agent. Removal and cloning of agents enables the organization to change in size.

The behavior of each aspectual agent is parameterized a variable, called its "eagerness", it influences the agent's behavior in its choice on whether to attack or not other agents. This parameter can be updated by the agent itself when it receives a recommendation from the morphological agents.

\subsection{The morphology}

To analyze the system, we chose to use only one characteristic of the agent's organizational state : its "supremacy". The idea of this measure is to relate the position of the agent within the organization : whether the agent is or not in a comfortable position. This is correlated to its energy level : the more energy the agent has, the more likely it is to survive. Hence, we chose to compute the agent's supremacy as equal to its energy level.

The shapes used to describe the organization's state are normalized and mean-centered histograms representing the agents' state distribution according to their supremacy. Histograms have the advantage of being easily comparable. It is possible to formulate a "reciprocal" of this mapping. An aspectual agent that is asked to change its vector value will try to do so by modified some of its variables (its eagerness) that alter its behavior accordingly.

\subsection{The analysis and control}

One analysis agent is used to control the system. This agent learns and classifies the histograms computed by the morphological agent. It can also directly know the actual number of agent in the aspectual organization, so it is able to determine, accordingly to rules defined by the system designer if the system is in a "good" or "bad" state and classify the corresponding shape properly (figure 1).

In this example, when the system behaves correctly there is no feedback. But when the population size is out of bounds, the analysis agent asks the morphological agent compute the appropriate feedback corresponding to the difference between the "good" and "bad" histograms.

\subsection{Results and discussion}

The limitation of space does not permit us to discuss in details the results of all the simulations but we will mention the essential.

Figure 2 sums up the results of two tests : one without control and the other with control. The instantaneous error rate is a pseudo-distance of the 


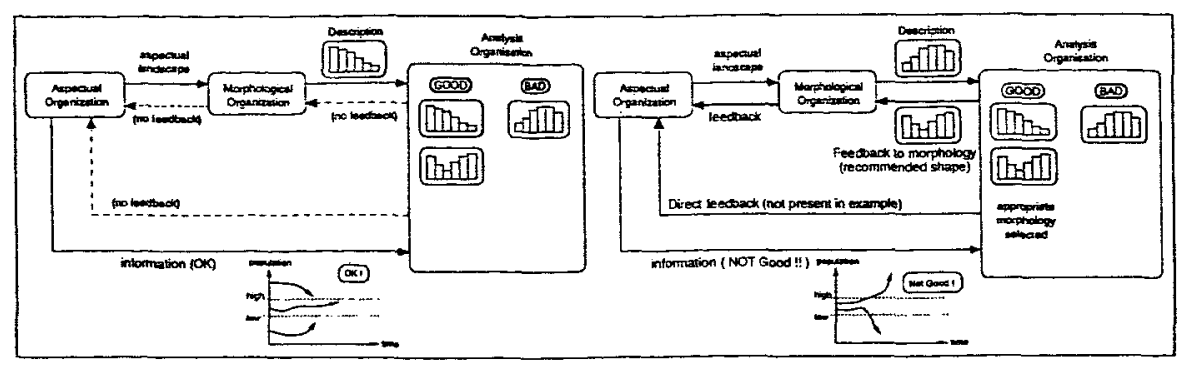

Figure 1: The left figure shows the system when it is considered as fine. The right figure shows when the system is needs adjustment.

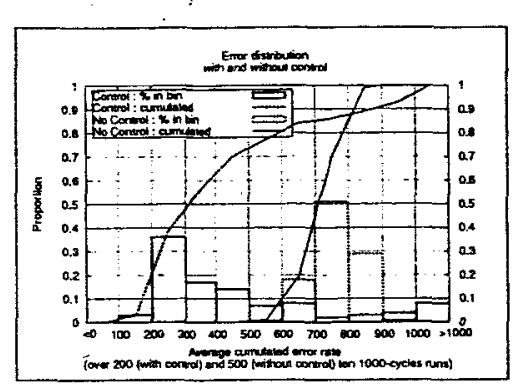

Figure 2: Comparative plot with and without control. Cumulative curves show that this system allows control in most cases (85\%).

population curve to the closest threshold (if the population curve is in-between both thresholds, the instantaneous error rate is zero). The error rate is the sum of all the instantaneous error rate in one 1000-cycles run. The average error rate for an analysis agent (if any) is computed over 10 such 1000 cycles run.

The reference test was done without control, it consisted of 500 tests. The other series of tests was done with control, over 200 tests (the difference of the number of tests is due to available time, the ones with control took longer to compute). In both cases the target population size was 50 agents with a margin of 0.2 (lower threshold and upper threshold are 40 and 60 respectively).

Figure 3 displays a couple of examples of the system's behavior, with and without control. These curves give a more palpable, qualitative, appreciation of control performance.

In most cases ( $85 \%$ ) the control improves the system's behavior. However, the histograms reveal that in $15 \%$ of the cases it does worse. One possible explanation is that, in some cases, the initial configuration of the aspectual organization (ie when the analysis agent has learned nothing yet) does not permit the analysis agent to "discover". adequate shapes, and then learns inappropriately, thus badly controlling the population size.. 


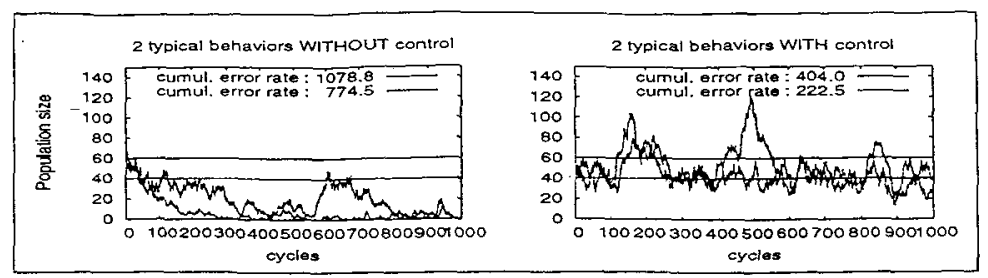

Figure 3: Example of typical (more than 50\% of the cases) system's behaviors (with and without control).

We also have noted that in some cases, only less than half of the aspectual agents needed to comply with the morphological injunctions, so that the population level was maintained at an appropriate value.

Developing more elaborated morphological analyzes (augmenting the aspectual vector with other aspects of the agent's behavior and using trajectories by introducing the time dimension in the morphological space) and using more appropriate learning mechanisms would allow finer control of the system.

\section{Conclusion}

We seek to develop a general method to analyze and control multi-agent system, and to make them self-adaptive. We briefly described the model based on the morphology approach of representing the system's state. This representation is available to the system in order to make it self-adaptive.

We illustrated the workings of the system with a simple example consisting. in population control. Shapes used in this example were histograms representing the relative distribution of one of the agent's properties. A simple learning mechanism permitted to outline and exploit a correlation between the micro-level behavior with macro-level behavior.

Other interests include developments of more elaborate morphology descriptions and understanding the needed properties of such description in order to be useful (toward formalization?).

\section{References}

1. Alain CARDON. Modéliser et concevoir une machine pensante. Approche constructible de la conscience artificielle. Éditions Automates Intelligents, march 2003.

2. F. CHANTEMARGUE, T. DAGAEFF, M. SCHUMACHER, and B. HIRSBRUNNER. The emergence of cooperation in a multiagent system, 1996.

3. Institut de Recherche en Informatique de Toulouse (IRIT). Théorie AMAS. http://www.irit.fr/SMAC/.

4. Catriona Mairi KENNEDY. Distributed reflective architectures for anomaly and autonomous recovery. PhD thesis, School of Computer Science, Faculty of Science, University of Birmingham, 2003.

5. Samuel LANDAU. Des $A G$ vers les $G A$. PhD thesis, Université de Paris VI, 2002. 